However, orientation-tuned cells in mice are not organized into columns, so the segregation between $\mathrm{ON}$ and OFF pathways may be independent of developing orientation maps. On the other hand, it is also possible that the non-overlapping $\mathrm{ON}$ and $\mathrm{OFF}$ inputs observed by Smith and Häusser ${ }^{1}$ may simply reflect the salt and pepper arrangement of RGCs in the mouse retina ${ }^{13}$ (Fig. 1a).

Why isn't the activity of neurons with shared subregions more correlated? One possibility is that these neurons encode different features of visual stimuli, such as orientation ${ }^{3}$. As orientation tuning in simple cells results from the relative positions of the $\mathrm{ON}$ and OFF subregions, the relative position between the two subregions can determine orientation selectivity of the cell. Smith and Häusser ${ }^{1}$ found that the average overlap of the second region was quite high (0.7) for neurons sharing one subregion. It will be important to determine whether such small shifts in relative subregion position can imbue these cells with substantially different orientation preferences, given the sharp orientation tuning observed in most cells $^{3}$. Alternately, the neuron pairs may have other receptive field differences that were not assessed by Smith and Häusser ${ }^{1}$, such as direction selectivity ${ }^{3}$.

Why is fine retinotopy not preserved in mouse V1? A key to interpreting the need for scatter may lie in the limitations imposed by the small size of the mouse visual cortex, where there is roughly one neuron for every RGC (Fig. 1a). For cortical cells to represent additional features of the visual stimulus, RGC inputs must be combined. However, because there are too few cortical cells in mouse V1, some information carried by the RGCs, such as fine retinotopy, may be lost (Fig. 1a). The observations of shared and spanned receptive fields suggest that the mouse visual system may sample portions of the visual field and pass them in parallel through several filters, extracting unique information, but introducing scatter into local retinotopy. Indeed, local scatter appears to be important in mouse auditory cortex (A1), where tonotopy fed forward from the thalamus is markedly disrupted, possibly in favor of more complex stimulus representations such as natural sounds ${ }^{14}$. It may be that functional scatter is a general principle in mouse sensory cortex, where discrete cell assemblies processing input from the same population of thalamic afferents act in a manner similar to a hypercolumn ${ }^{4}$ (Fig. 1a).

The situation is likely very different in visual systems of other model species, such as cats and macaques, which are more similar to humans. Because these species have about 100 cells in V1 for every RGC with each receptive field composed of inputs from many RGCs (Fig. 1b), such marked tradeoffs between retinotopy and diversity may not be necessary. Indeed, the organization of orientation tuning in cat V1 is smooth even at single-cell resolution ${ }^{15}$. It will be important to determine whether retinotopy is equally smooth. Notably, some evidence suggests that maps of orientation and retinotopy may be interrelated ${ }^{5}$. The microscopic relationship between the two maps may have important implications for the principles governing the organization and development of the cortical microcircuit ${ }^{13}$. Thus, it will be important to examine how maps of retinotopy and orientation are related in these species on microscopic scales.

By zooming into the mouse visual system with a two-photon microscope, Smith and Häusser ${ }^{1}$ provide important insights into how cortex produces diverse functional tuning under the constraint of low divergence. Their work will serve as a valuable reference for imaging the visual system at finer resolution in other species. In these systems, their powerful technique could be used to systematically study how cortical representations of visual features, such as orientation and direction of motion, interact with retinotopy. At the same time, developing our understanding of rodent V1 (refs. 3,7) sets up a system for studying visual processing that is amenable to the full spectrum of genetic and imaging techniques. Because of its accessibility, future work in this system will likely produce breakthroughs in our understanding of the general principles underlying cortical processing. Imagine what lies ahead.

\section{COMPETING FINANCIAL INTERESTS}

The authors declare no competing financial interests.

1. Smith, S.L. \& Häusser, M. Nat. Neurosci. 13, 1144 1149 (2010).

2 Ohki, K., Chung, S., Ch'ng, Y.H., Kara, P. \& Reid, R.C. Nature 433, 597-603 (2005).

3 Niell, C.M. \& Stryker, M.P. J. Neurosci. 28, 7520 7536 (2008).

4. Hubel, D.H. \& Wiesel, T.N. Proc. R. Soc. Lond. B Biol. Sci. 198, 1-59 (1977).

5. Das, A. \& Gilbert, C.D. Nature 387, 594-598 (1997).

6. DeAngelis, G.C., Ghose, G.M., Ohzawa, I. \& Freeman, R.D. J. Neurosci. 19, 4046-4064 (1999).

7 Yoshimura, Y., Dantzker, J.L. \& Callaway, E.M. Nature 433, 868-873 (2005).

8 Jin, J.Z. et al. Nat. Neurosci. 11, 88-94 (2008).

9. Zahs, K.R. \& Stryker, M.P. J. Neurophysiol. 59, 14101429 (1988).

10. Miller, K.D. Neuroreport 3, 73-76 (1992).

11. Miller, K.D. J. Neurosci. 14, 409-441 (1994).

12. Ringach, D.L. J. Neurophysiol. 92, 468-476 (2004).

13. Ringach, D.L. PLoS One 2, e251 (2007).

14. Castro, JB. \& Kandler, K. Nat. Neurosci. 13, 271-273 (2010).

15. Ohki, K. et al. Nature 442, 925-928 (2006).

\title{
Building the preBötzinger complex
}

The preBötzinger complex (preBötC) is a critical component of the respiratory rhythmgenerator that underlies mammalian breathing. However, we know little about the developmental origins of its neurons or the molecular mechanisms that determine their fate. Anatomically, it is characterized as a collection of glutamatergic interneurons that express the neurokinin type1 receptor (NK1R) and somatostatin (Sst). These glutamatergic interneurons are rhythmically active and are indispensable for the generation of the respiratory rhythm. They also project across the midline, connecting the two halves of the preBötC to establish bilateral synchrony, which is critical for efficient breathing.

On page 1066, Bouvier and colleagues identified the homeobox gene $D b x 1$ as being critical

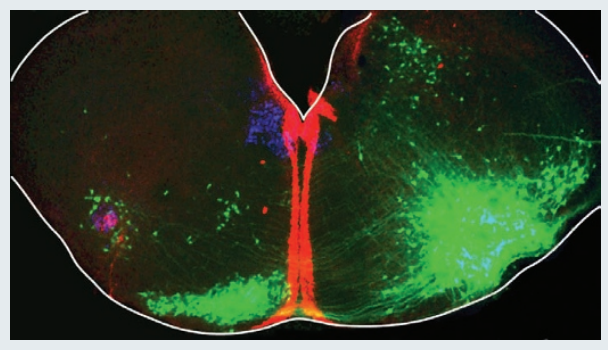
for controlling the fate of these glutmatergic interneurons. Using a genetic strategy, supported by molecular and electrophysiological techniques, they found that Dbx1-expressing progenitors give rise to the glutamatergic interneurons that are necessary for rhythm generation and bilateral synchrony of the preBötC, including the constitutive NK1R- and Sst-expressing neurons, and that $D b x 1$ is critical for establishing their fate during embryonic development. Moreover, they found that the establishment of commissural connections between the halves of the preBötC requires expression of the Robo3 receptor by the Dbx1-derived interneurons. A coronal section of the mouse embryonic medulla is shown in which commissural connections are made by biocytin-labeled interneurons (green) projecting to the vicinity of the contralateral NK1R-positive neurons of the preBötC (red) near the nucleus ambiguus (labeled blue).

Kathleen A Dave 